Daniela Basso, Ada Aita, Filippo Navaglia, Elisa Franchin, Paola Fioretto, Stefania Moz, Dania Bozzato, Carlo-Federico Zambon, Barbara Martin, Chiara Dal Prà, Andrea Crisanti and Mario Plebani*

\title{
SARS-CoV-2 RNA identification in nasopharyngeal swabs: issues in pre-analytics
}

https://doi.org/10.1515/cclm-2020-0749

Received May 19, 2020; accepted May 24, 2020; published online June 23,2020

\begin{abstract}
Objectives: The direct identification of SARS-CoV-2 RNA in nasopharyngeal swabs is recommended for diagnosing the novel COVID-19 disease. Pre-analytical determinants, such as sampling procedures, time and temperature storage conditions, might impact on the end result. Our aim was to evaluate the effects of sampling procedures, time and temperature of the primary nasopharyngeal swabs storage on real-time reverse-transcription polymerase chain reaction (rRT-PCR) results.
\end{abstract}

Methods: Each nasopharyngeal swab obtained from 10 hospitalized patients for COVID-19 was subdivided in 15 aliquots: five were kept at room temperature; five were

*Corresponding author: Professor Mario Plebani, MD, Department of Medicine - DIMED, University of Padova, Padova, Italy; Department of Laboratory Medicine, University Hospital of Padova, Padova, Italy, E-mail: mario.plebani@unipd.it. https://orcid.org/0000-0002-02701711

Daniela Basso, Ada Aita, Stefania Moz, Dania Bozzato and CarloFederico Zambon: Department of Medicine - DIMED, University of Padova, Padova, Italy; Department of Laboratory Medicine, University Hospital of Padova, Padova, Italy, E-mail: daniela.basso@unipd.it (D. Basso), ada.aita@unipd.it (A. Aita), stefania.moz@unipd.it

(S. Moz), dania.bozzato@unipd.it (D. Bozzato),

carlofederico.zambon@unipd.it (C.F. Zambon)

Filippo Navaglia: Department of Laboratory Medicine, University Hospital of Padova, Padova, Italy,

E-mail: filippo.navaglia@aopd.veneto.it

Elisa Franchin: Department of Molecular Medicine - DMM, Microbiology and Virology, University of Padova, Padova, Italy, E-mail: elisa.franchin@unipd.it

Paola Fioretto, Barbara Martin and Chiara Dal Prà: Department of Medicine - DIMED, Internal Medicine 3, University of Padova, Padova, Italy, E-mail: paola.fioretto@unipd.it (P. Fioretto), barbara.martin@aopd.veneto.it (B. Martin), chiara.dalpra@aopd.veneto.it (C. Dal Prà)

Andrea Crisanti: Department of Molecular Medicine - DMM Microbiology and Virology, University of Padova, Padova, Italy; Department of Life Sciences, Imperial College London, South Kensington Campus, London, UK, E-mail: andrea.crisanti@unipd.it refrigerated $\left(+4^{\circ} \mathrm{C}\right)$; five were immediately mixed with the extraction buffer and refrigerated at $+4^{\circ} \mathrm{C}$. Every day and for 5 days, one aliquot per condition was analyzed (rRT-PCR) for SARS-CoV-2 gene E and RNaseP and threshold cycles (Ct) compared. To evaluate manual sampling, 70 nasopharyngeal swabs were sampled twice by two different operators and analyzed separately one from the other.

Results: A total of 6/10 swabs were SARS-CoV-2 positive. No significant time or storage-dependent variations were observed in SARS-CoV-2 Ct. Re-sampling of swabs with SARS-CoV-2 Ct lower than 33 resulted in highly reproducible results $(\mathrm{CV}=2.9 \%)$, while a high variability was observed when $\mathrm{Ct}$ values were higher than 33 ( $\mathrm{CV}=10.3 \%$ ). Conclusions: This study demonstrates that time and temperature of nasopharyngeal swabs storage do not significantly impact on results reproducibility. However, swabs sampling is a critical step, and especially in case of low viral load, might be a potential source of diagnostic errors.

Keywords: COVID-19; nasopharyngeal swab; pre-analytical; SARS-CoV-2.

\section{Introduction}

The COVID-19 pandemic poses several questions concerning the diagnosis of symptomatic, but mainly of asymptomatic, subjects, who represent a potential, hidden source of viral dissemination [1]. Identifying and isolating these so-called asymptomatic carriers, and tracing all their potential contacts, together with ensuring social distancing and behavioral change in the population, are considered strategies of crucial importance in limiting further viral spread [2]. The available armamentarium for the diagnosis of COVID-19 includes the direct search for viral SARS-CoV-2 RNA sequences in nasopharyngeal swabs, as well as the detection of serum or blood antibodies by means of immunometric or rapid immunochromatographic assay [3-6]. An increase in antibodies in blood/serum usually occurs 1-2 weeks after the onset of symptoms [7, 8], but viral transmission from one individual to another is estimated to occur not only concurrent with the symptomatic window, but also during the incubation period, which ranges from one to two, or even 
more, weeks before any clinical signs of the disease become evident $[1,9]$. Therefore, the identification of asymptomatic carriers and those with recent onset disease must be based on the direct identification of viral RNA, serology being useful as a complementary approach for disease diagnosis and monitoring, and for epidemiological studies [7, 10].

The direct identification of viral RNA in nasopharyngeal swab samples is the first choice for diagnosis, which, as recommended by the World Health Organization (WHO), should be timely and accurate $[4,11]$. However, neither of these two requirements, i.e., timely identification of viral RNA, is necessarily met in situations of the high testing demand occurring in the pandemic context, especially in view of potential limitations in the supply of reagents. Moreover, despite the availability of high-throughput platforms for RNA analysis based on real-time reversetranscription polymerase chain reaction (rRT-PCR) methodology, such as the fully integrated and automated Cobas 6800 system that allows a respectably high number of specimens tested per day (about 1200), many laboratories perform rRT-PCR molecular testing using separate instruments for nucleic acid extraction and rRT-PCR that are relatively time consuming and cause human resources deployment $[4,9,12]$. Despite the high sensitivity of molecular methods, the diagnostic sensitivity of viral RNA identification in nasopharyngeal swabs does not exceed $70 \%[9,13,14]$. In the complex and unexpected situation that the world faced at the beginning of 2020, it is hardly surprising that some late testing occurred. Yet any delay in the total testing process not only delays diagnosis, but also represents a potential cause of diagnostic error, mainly due to false-negative results consequent to RNA degradation.

In laboratory medicine, the well-known relevance of the pre-analytical phase as a determinant of the overall accuracy of results appears to be of outmost importance also in the field of COVID-19 molecular diagnosis [15-17]. Among the pre-analytical factors to be considered, collection method (type of swab), appropriate training of the collecting individual, use of "dry" swabs or of transport medium are very important issues. The severely limited availability of reagents, including viral transport media (VTM), prompted researchers to evaluate whether saline or phosphate-buffered saline (PBS) might safely replace standard VTM or universal transport media (UTM) $[18,19]$. PBS was demonstrated to preserve nasopharyngeal swab samples up to $18 \mathrm{~h}$ at room temperature and recommended by the FDA in the absence of VTM/UTM (https://www.fda. gov/medical-devices/emergency-situations-medical-devices/ faqs-testing-sars-cov-2; last accessed 22 May 2020).
However, transport mode, time lapse and storage temperature between nasopharyngeal specimen collection and laboratory analysis are also relevant, since they might determine the activation of molecular degradation processes, RNA being one of the most sensitive molecules.

The analysis of nasopharyngeal swabs, moreover, calls for some initial manual steps, such as sampling, which entail a risk of error that impacts on the end result.

The end points of this study were to evaluate whether primary nasopharyngeal swabs, after prolonged storage at different temperatures or after being aliquoted in nucleic acid extraction buffer, allow reproducible rRT-PCR results, and whether inter-individual labor in primary tube sampling might impact on the reproducibility of results.

\section{Materials and methods}

A total of 10 patients ( 6 males, 4 females, age range 51- 87) enrolled for the study were hospitalized for COVID-19 infection in a semi-intensive care unit. After collection of fully informed consent (Local Ethic Committee Nr. 27444), two replicated nasopharyngeal swabs using the $\sum$-VCM (Medical Wire \& Equipment, UK) were obtained from each patient. This commercial swab type comprises a self-standing conical based vial with $3 \mathrm{~mL}$ of universal transport Virocult ${ }^{\circledR}$ medium (VCM) allowing viruses transport and identification by both gold standard cell culture methods or by molecular techniques. It was chosen because of the large volume of viral transport medium, which allows multiple testing. The two swabs obtained from each patient were pooled, mixed and then subdivided into 15 aliquots within $2 \mathrm{~h}$ from specimen collection, during which nasopharyngeal swabs were maintained refrigerated at $4{ }^{\circ} \mathrm{C}$. The aliquots, $300 \mu \mathrm{L}$ each, were transferred into $2 \mathrm{~mL}$ tubes (Sarstedt AG \& Co., Germany). Five aliquots were kept at room temperature, five were refrigerated $\left(+4^{\circ} \mathrm{C}\right)$ and five were immediately mixed (3:1, v:v) with Nuclisens ${ }^{\circledast}$ easyMAG ${ }^{\circledast}$ Extraction buffer 1 (bioMérieux Diagnostics, Italy) and then stored at $+4^{\circ} \mathrm{C}$. One aliquot of each patient for each storage condition was analyzed every day for 5 days, the analyses being started $24 \mathrm{~h}$ after collection (day 1). Any of the two nasopharyngeal swabs aliquots, one stored at room temperature and another refrigerated, were mixed (3:1, v:v) with Nuclisens $^{\circledast}$ easyMAG ${ }^{\circledast}$ Extraction buffer 1 immediately before RNA extraction. This procedure was chosen to lyse viral particles before loading samples on the automated platform for RNA extraction to further enhance safety for operators. Every day, from day 1 to day 5 , the aliquots of all patients were analyzed in batch: RNA was extracted using an automated platform (Magna Pure 96 Instrument, Roche Diagnostics, USA) and then used for rRT-PCR, which was performed according to Corman et al. [20] by ABI prism ${ }^{\circledR}$ AB 7900 or $7900 \mathrm{HT}$ or QuantStudio $^{\mathrm{TM}} 5$ Real-Time PCR Systems (Applied Biosystems, USA). The performance of any system was monitored by two internal positive and four negative quality controls (IQC) that were always run in parallel with patients' samples. In brief, the rRT-PCR was performed in separate reactions with SARS-CoV-2 gene $\mathrm{E}$ and RNaseP analyses. In the absence of amplification of the latter target, the analysis was considered invalid, and this criterion was used for repeat testing. The threshold cycle (Ct) of SARS-CoV-2 gene E and of RNaseP was obtained after standardization of the rRT-PCR instruments software settings as 
follows: baseline calculated in the cycle range 3-15; fixed threshold at 0.2. The $\mathrm{Ct}$ of each analysis was considered for data comparison. In each analytical set, two negative and two positive controls were always run in parallel with samples. The positive controls (IQC) were RNA obtained from positive samples that were stored at $-80^{\circ} \mathrm{C}$ for a maximum of 1 week.

The statistical analysis of data was made by Repeated measures ANOVA, Tukey's multiple comparison test, One-way ANOVA, Student's t-test for paired and unpaired data using STATA 13.1 statistical software (StataCorp, 4905 Lakeway Drive, USA).

\section{Results}

The 10 nasopharyngeal swabs studied resulted SARS-CoV2 positive in six and negative (no amplification signal after 45 cycles) in four cases. Figure 1 shows the $\mathrm{Ct}$ variations of SARS-CoV-2 gene E and of the RNaseP observed for each patient over time in the three different storage conditions. With respect to SARS-CoV-2, no significant time-dependent or condition-dependent variation of the $\mathrm{Ct}$ was found (repeated measures analysis of variance: $\mathrm{F}=1.291, \mathrm{p}=0.284$ for time and $\mathrm{F}=0.0162, \mathrm{p}=0.984$ for condition, $\mathrm{F}=1.602$, $\mathrm{p}=0.144$ for interaction). However, some data points showed higher $\mathrm{Ct}$ values compared to their flanking data. This phenomenon occurred after 3 days of storage.

RNaseP varied over time and by condition $(\mathrm{F}=4.6011$, $\mathrm{p}=0.0018$ for time and $\mathrm{F}=2.903, \mathrm{p}=0.0721$ for condition, $\mathrm{F}=2.393, \mathrm{p}=0.0205$ for interaction): a significant difference was found between swabs maintained at room temperature and swabs mixed soon after collection with extraction buffer and stored at $+4{ }^{\circ} \mathrm{C}$ after days four and five $(\mathrm{p}<0.05$ Tukey's multiple comparison test). Table 1 shows the results obtained on calculating the coefficient of variations (CV) of the measured $\mathrm{Ct}$ for any individual sample in the three different experimental conditions. For both SARSCoV-2 gene $\mathrm{E}$ and RNaseP, no significant differences in coefficients of variation were found between the different storage conditions investigated.

Since the overall 5-day SARS-CoV-2 gene E and RNaseP coefficients of variation reflected the influence of preanalytical storage and analytical variability, to further understand the impact of pre-analytical variability, we compared SARS-CoV-2 gene $\mathrm{E}$ and RNaseP Ct obtained on the same day (i.e., after in-batch RNA extraction and realtime PCR analysis); Figure 2 shows the results obtained. No statistically significant results were found at repeated measures ANOVA for SARS-CoV-2 gene $\mathrm{E} \quad(\mathrm{F}=0.41$, $\mathrm{p}=0.6761$ day $1 ; \mathrm{F}=2.09, \mathrm{p}=0.1744$ day 2; $\mathrm{F}=1.86$; $\mathrm{p}=0.2055$ day $3 ; \mathrm{F}=0.68, \mathrm{p}=0.5268$ day $4 ; \mathrm{F}=0.65$, $\mathrm{p}=0.5448$ day 5$)$, unlike for RNAse $P(\mathrm{~F}=3.91, \mathrm{p}=0.0390$ day $1 ; \mathrm{F}=7.62, \mathrm{p}=0.0040$ day $2 ; \mathrm{F}=5.05, \mathrm{p}=0.0181$ day $3 ; \mathrm{F}=12.69$, $\mathrm{p}=0.0004$ day 4; $\mathrm{F}=5.56, \mathrm{p}=0.0132$ day 5). Significantly lower $\mathrm{Ct}$ were found in samples stored in extraction buffer at $4{ }^{\circ} \mathrm{C}$ after two (Bonferroni adjusted $\mathrm{p}$-value $=0.0276$ ), three $(\mathrm{p}=0.0348)$, four $(\mathrm{p}=0.004)$ and five $(\mathrm{p}=0.0072)$ days with respect to swabs stored at $4^{\circ} \mathrm{C}$.

Analytical intra-laboratory variability of rRT-PCR was evaluated by means of repeat analyses of two positive RNA samples, run for 7 days in the same experimental period. Table 2 shows the results obtained.

\section{SARS-CoV2 gene E}

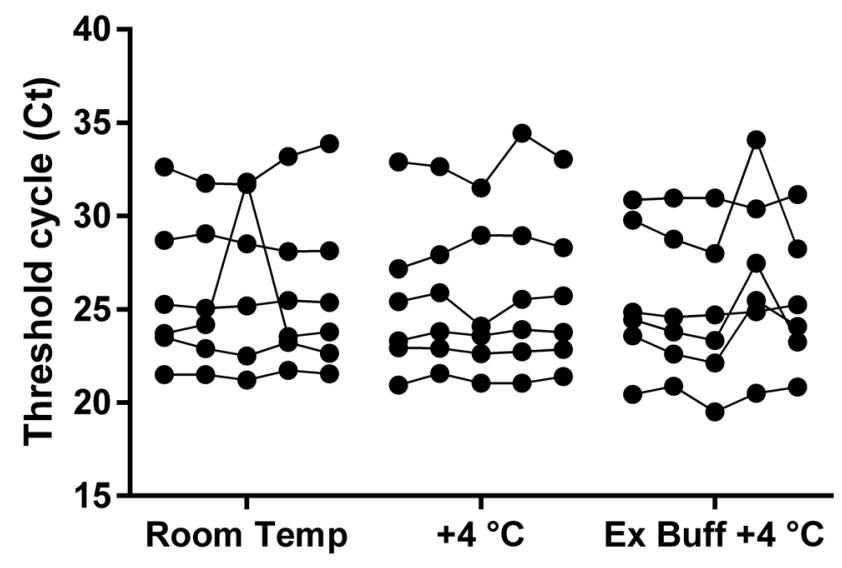

RNAseP

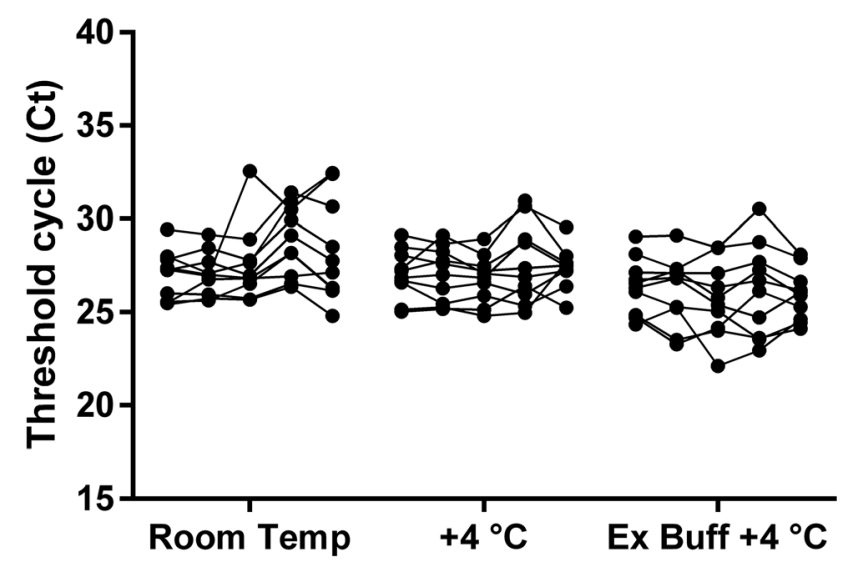

Figure 1: The effects of storage temperature and duration on SARSCoV-2 gene $E$ and RNaseP results.

The upper panel shows the threshold cycles (Ct) of 6/10 SARS-CoV-2 gene $\mathrm{E}$ positive swabs stored at room temperature (Room Temp), at $+4{ }^{\circ} \mathrm{C}$ and at $+4^{\circ} \mathrm{C}$ after mixing (3:1, v:v) with extraction buffer (Ex Buff $+4^{\circ} \mathrm{C}$ ). Each line represents replicated values obtained from one individual sample on subsequent days (i.e., days $1-5$ ). The lower panel shows the threshold cycles $(\mathrm{Ct})$ of RNaseP of the 10 swabs analyzed, comprising six positive and four negative SARS-CoV-2 cases, following storage in the conditions reported in the upper panel. 
Table 1: Time and temperature-dependent coefficients of variation (CV).

\begin{tabular}{|c|c|c|c|c|}
\hline & $\begin{array}{l}\text { Room temperature mean } \\
\text { CV } \% \pm \text { SD (CV \% range) }\end{array}$ & $\begin{array}{r}+4^{\circ} \mathrm{C} \text { mean CV \% } \\
\pm \mathrm{SD}(\mathrm{CV} \% \text { range })\end{array}$ & $\begin{array}{r}\text { Extraction buffer }+4^{\circ} \mathrm{C} \text { mean } \\
\text { CV } \% \pm \mathrm{SD}(\mathrm{CV} \% \text { range })\end{array}$ & One-way ANOVA \\
\hline $\begin{array}{l}\text { SARS-CoV-2 } \\
\quad \text { gene } E(n=6)\end{array}$ & $\begin{array}{r}3.62 \pm 5.21 \\
(0.63-14.13)\end{array}$ & $\begin{array}{l}1.91 \pm 1.10 \\
(0.58-3.19)\end{array}$ & $\begin{array}{l}4.96 \pm 3.70 \\
(1.62-4.43)\end{array}$ & $\begin{array}{r}F=1.00, \\
p=0.3918\end{array}$ \\
\hline RNase $P(n=10)$ & $\begin{array}{l}3.43 \pm 2.39 \\
(0.71-8.06)\end{array}$ & $\begin{array}{l}2.47 \pm 1.09 \\
(1.03-4.43)\end{array}$ & $\begin{array}{l}2.76 \pm 1.24 \\
(0.96-4.81)\end{array}$ & $\begin{array}{r}F=0.87, \\
p=0.4309\end{array}$ \\
\hline
\end{tabular}

For any individual sample, the rRT-PCR threshold cycle (Ct) obtained in the 5 days of replicated measurements of SARS-CoV-2 gene E and RNaseP were considered in order to calculate the coefficient of variation (CV). For every condition, mean values \pm standard deviation (SD) and range were calculated, and compared statistically (one-way ANOVA).

Confirmation of doubtful results might call for a second round of rRT-PCR analysis, which, in our routine series was repeated in $57 / 10,350(0.55 \%)$ cases by re-sampling the nasopharyngeal swabs. The samples were analyzed twice due to: (1) amplification curves without a perfect exponential kinetic associated with a Ct higher than $33(n=48)$; (2) observation of an exponential kinetic amplification curve not exceeding the 0.2 threshold ( $\mathrm{n}=9$ ). Twenty-seven of the 57 re-analyzed samples presented no amplification signal at the second round and were classified as negative, while the remaining 30 samples presented an exponential amplification kinetic. In the 27 samples found to be negative at the second analysis, the $\mathrm{Ct}$ of the first real-time PCR analysis was higher $(38.44 \pm 2.82$, mean \pm SD) than in the 30 samples confirmed as positive at the second analysis $(36.23 \pm 2.31)$ (Student's t-test for unpaired data: $t=3.0145$, $\mathrm{p}<0.005)$. For the 30 samples with a measurable $\mathrm{Ct}$ at both the first and the second rRT-PCR, mean values with SD were

\section{SARS-CoV-2 gene E}

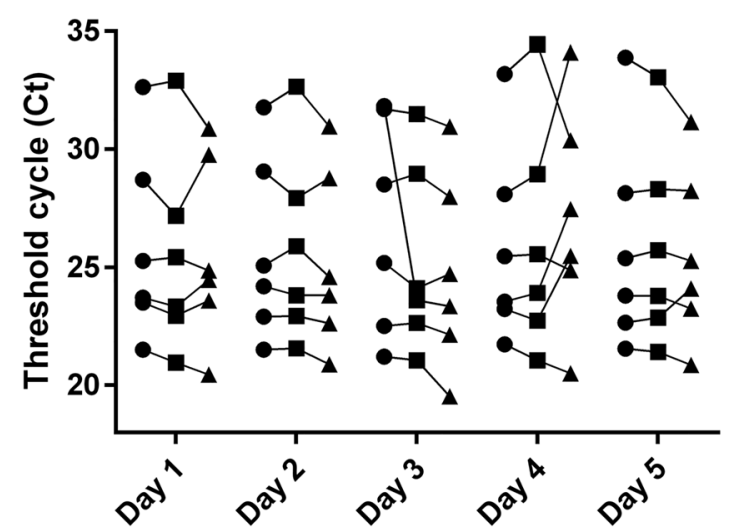

- Room Temperature

Refrigerated $+4^{\circ} \mathrm{C}$

$\leftarrow$ Extraction buffer $+4{ }^{\circ} \mathrm{C}$

\section{RNAseP}

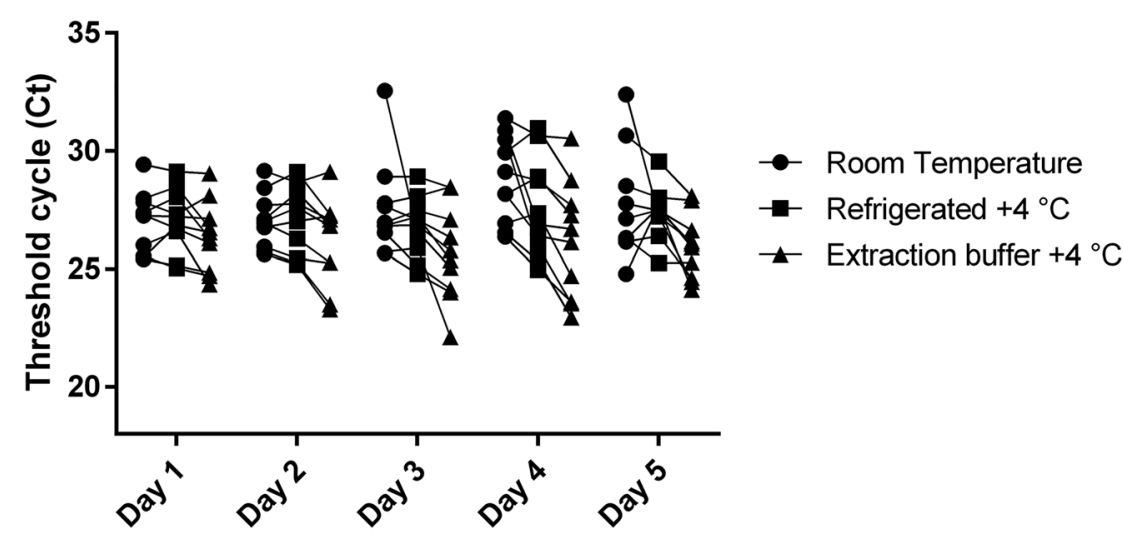

Figure 2: The effects of storage temperature and duration on SARS-CoV-2 gene $E$ and RNaseP results.

The upper panel shows the threshold cycles (Ct) of $6 / 10$ SARS-CoV-2 gene $E$ positive swabs stored at room temperature, at $+4{ }^{\circ} \mathrm{C}$ and at $+4^{\circ} \mathrm{C}$ after mixing (3:1, v:v) with extraction buffer. Each line represents values obtained from one individual sample on the same day. The lower panel shows the threshold cycles (Ct) of RNaseP of the 10 swabs analyzed, comprising six positive and four negative SARS-CoV-2 cases, following storage in the conditions reported in the upper panel. 
Table 2: Analytical variability.

\begin{tabular}{|c|c|c|c|c|c|c|c|}
\hline & & IQC $1(n=20)$ & IQC $2(n=20)$ & IQC $3(n=21)$ & IQC $4(n=21)$ & IQC $5(n=18)$ & IQC $6(n=18)$ \\
\hline SARS-CoV-2 & Mean $\mathrm{Ct} \pm \mathrm{SD}$ & $18.68 \pm 0.26$ & $23.81 \pm 0.39$ & $16.65 \pm 0.36$ & $22.08 \pm 0.43$ & $28.80 \pm 0.51$ & $27.81 \pm 0.58$ \\
\hline gene $\mathrm{E}$ & CV \% & 1.40 & 1.65 & 2.18 & 1.96 & 1.77 & 2.09 \\
\hline \multirow[t]{2}{*}{ RNAseP } & Mean $\mathrm{Ct} \pm \mathrm{SD}$ & $21.85 \pm 0.25$ & $24.88 \pm 0.31$ & $22.46 \pm 0.47$ & $22.07 \pm 0.34$ & $22.53 \pm 0.36$ & $28.88 \pm 0.32$ \\
\hline & CV \% & 1.14 & 1.26 & 2.07 & 1.54 & 1.61 & 1.09 \\
\hline
\end{tabular}

Mean values, standard deviation (SD) and coefficients of variation (CV) of the Ct of SARS-CoV-2 gene E and RNaseP obtained after replicated analyses of positive samples (IQC) are reported. Each IQC was stored for a maximum of one week at $-80^{\circ} \mathrm{C}$.

calculated and plotted (Figure 3, upper panel). For purposes of comparison, a series of 14 positive samples with a Ct lower than 33 were re-tested following the abovedescribed same procedure. The results obtained (mean
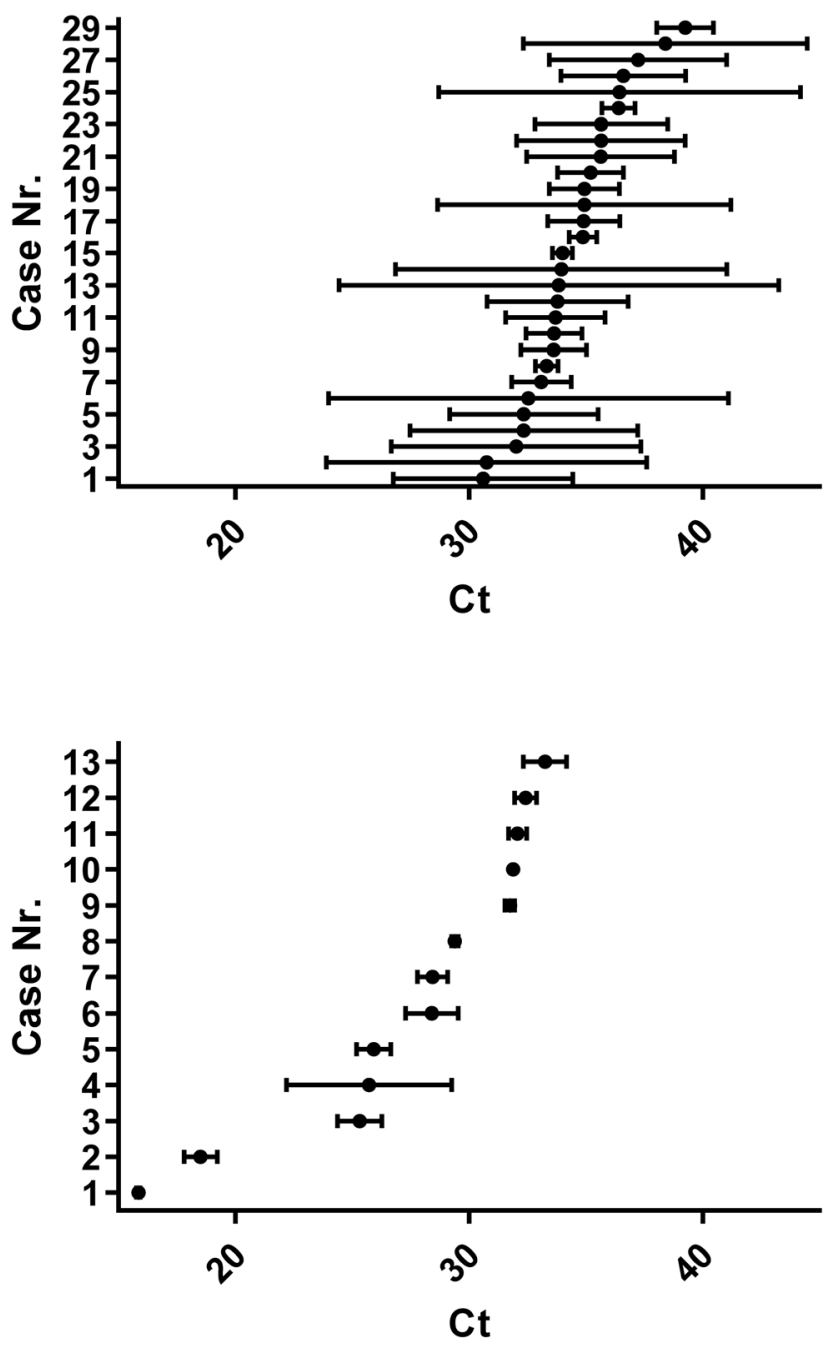

Figure 3: Operator-dependent pre-analytical variability. Mean values (points) with standard deviations (bars) of rRT-PCR Ct values obtained after replicated nasopharyngeal swabs sampling by two different operators in subsequent days.

The upper panel shows data from swabs with a first $\mathrm{Ct}$ value higher than 33, while in the lower panel data from swabs with a first $\mathrm{Ct}$ lower than 33 are reported. values with standard deviation of the two replicated analyses) are shown in Figure 3 (lower panel). In the attempt to ascertain whether nasopharyngeal sampling errors might impact on rRT-PCR results, two nasopharyngeal samples presenting Ct results of 36 and 38, respectively, were newly sampled three times: without vortexing, after vortexing and after vortexing with a sampling volume reduced (from 300 to $100 \mu \mathrm{L}$ ). The results obtained are shown in detail in Table 3.

\section{Discussion}

rRT-PCR or loop-mediated isothermal amplification (RTLAMP) in patients with suspected COVID-19 using nasopharyngeal swabs allows accurate diagnosis in the presence of the infection [9, 21]. Among the various SARS-CoV-2 gene targets that include RNA dependent-RNA polymerase, spike (S) and nucleocapsid (N) genes, the one-step identification of the envelope (E) gene is sensitive and specific $[9,12,20]$. When $\mathrm{rRT}-\mathrm{PCR}$ for SARS-CoV-2 gene $\mathrm{E}$ is performed, the detection of viral RNA is based on exponential amplification kinetics, the threshold amplification cycle $(\mathrm{Ct})$ being correlated with the copy numbers, or viral load present in the sample [21]. rRT-PCR, however, is only one of the multiple pre-analytical and analytical steps that ultimately lead to the final result. These steps can be summarized as follows: (1) collecting nasopharyngeal swab; (2) transporting the sample to the laboratory; (3) sampling the nasopharyngeal swab and transferring it into a tube containing the RNA extraction buffer; (4) extracting RNA; (5) rRT-PCR; and (6) interpreting data. Variability occurring in any of these steps might impact on the final result. The first source of pre-analytical variability is specimen collection, potentially affected by personnel training, swab type and transport media. The preferred specimen is a nasopharyngeal swab placed in viral transport media, although in times of supplies limitations, PBS or even saline could be alternative media $[18,19]$. In the present study, nasopharyngeal swabs collected by trained nurses from 10 COVID-19 patients were used to evaluate the 
Table 3: Operator-independent pre-analytical variability.

\begin{tabular}{|c|c|c|c|c|c|c|}
\hline & \multirow{2}{*}{$\begin{array}{r}\text { First rRT-PCR } \\
\text { Ct values }\end{array}$} & & & & \multicolumn{2}{|c|}{ Second rRT-PCR Ct values } \\
\hline & & $\begin{array}{l}\text { Sampling } 300 \mu \mathrm{L} \\
\text { without vortexing }\end{array}$ & $\begin{array}{r}\text { Sampling } 300 \mu \mathrm{L} \\
\text { after vortexing }\end{array}$ & $\begin{array}{r}\text { Sampling } 100 \mu \mathrm{L} \\
\text { after vortexing }\end{array}$ & Mean $\mathrm{Ct} \pm$ SD & CV \% \\
\hline Patient 1 & 36 & 33.47 & 33.85 & 33.75 & $33.69 \pm 0.28$ & $0.83 \%$ \\
\hline Patient 2 & 38 & 35.73 & 36.31 & 37.72 & $36.59 \pm 1.02$ & $2.79 \%$ \\
\hline
\end{tabular}

Two naso-pharyngeal swabs with high Ct for SARS-CoV-2 gene E were re-sampled $24 \mathrm{~h}$ after the first rRT-PCR by the same operator, who followed three different manual procedures: (1) $300 \mu \mathrm{L}$ sampled without previous vortexing; (2) $300 \mu \mathrm{L}$ sampled after vortexing; $100 \mu \mathrm{L}$ sampled after vortexing. Coefficients of variation (CV) were calculated from data of the three replicated values obtained at the second rRT-PCR.

impact of storage temperature and time, to mimic one of the field conditions of the pre-analytical phase, known to be a greater source of variability than the analytical phase [15-17]. In particular, Lippi and Colleagues summarized potential pre-analytical and analytical vulnerabilities in COVID-19 molecular diagnosis and suggested practical indications to minimize the risk of diagnostic errors [17]. Transport of samples to the laboratory and storage before sampling might entail a prolonged time interval, especially when only few reference laboratories are authorized to perform the analyses and the sample should be transported between institutions or to a reference laboratory. In this context, it is important to know whether strict temperature control is required in order to guarantee accurate results [21]. Our experiments demonstrate that reproducible results can be obtained until the $5^{\text {th }}$ day of sample taking not only when primary nasopharyngeal swab samples are maintained at $+4^{\circ} \mathrm{C}$, but also when they are kept at room temperature, although refrigeration of the primary sample allows the highest levels of reproducibility. Although during the 5 experimental days some points showed a slight $\mathrm{Ct}$ increase, suggesting a potential decay of the viral molecular signal, no variation was observed in any of the studied conditions within the first $48 \mathrm{~h}$. Our findings could be translated in the field condition with two levels of recommendations: (1) Transport and storage of nasopharyngeal swabs in viral transport media at room temperature for a period of up to $48 \mathrm{~h}$ prior rRT-PCR is highly reliable; (2) For longer periods and up to 5 days, refrigeration of the sample is preferable. Storage of samples might be necessary also after specimens reach the laboratory, which might suffer from some delay in testing for high testing demand. The addition of RNA extraction buffer to the sample upon arrival to the laboratory might maximize the preservation of RNA molecules. In agreement with this, in the time frame of 5 days, we found that the refrigerated storage of samples in RNA extraction buffer is not only safe, but resulted also in lower $\mathrm{Ct}$ values, confirming that the extraction buffer itself maximizes the preservation of RNA molecules [22].
Our findings also show that a potentially relevant pre-analytical source of variability is nasopharyngeal swab sampling when the $\mathrm{Ct}$ is higher than 33 (i.e., when a low viral load is expected). Indeed, in our series of repeated analyses of samples with a mean $\mathrm{Ct}$ of 36.23, the coefficient of variation ranged from 1 to $28 \%$ (mean value, $10 \%$ ), whereas repeated sampling of primary nasopharyngeal swabs with a lower mean Ct value (27.61) allowed more reproducible results with coefficients of variation ranging from 0 to $14 \%$ (mean value, $2.88 \%$ ). This suggests that the manual workup required at the beginning of sample processing might be a relevant source of variability in the presence of a low viral load, in accordance with previous findings in clinical studies reporting high false negative rates in asymptomatic patients, and fluctuating rRT-PCR results in COVID-19 hospitalized patients [13]. To further verify the impact of sampling in cases of low viral load, two samples from patients with high Ct values were selected and sampled three times by the same operator, who respected or intentionally disregarded the standard recommended procedure for vortexing nasopharyngeal swabs before sampling. Unexpectedly, the results for both the analyzed samples were highly reproducible. The differences between the first and the second evaluation set-up were as follows: the first series of experiments with repeated sampling were performed on two subsequent days by different operators and the samples were analyzed in different analytical series; the second series of experiments undertaken on the limited series of two samples were performed by one operator and analyzed in batch in the same analytical run. One might therefore ask whether variability in re-testing samples is operator- or analysis-dependent. We feel it is reasonable to rule out the latter hypothesis since the intra-laboratory (i.e., day by day) imprecision was lower than $3 \%$, thus supporting the robustness of rRT-PCR analysis. On the contrary, we support the hypothesis that inter-operator variability might be one of the main causes of variability. Another potential cause of variability, however, might be related 
to the nasopharyngeal swab types used, some of which are problematic to use for sampling, while others are more user friendly.

The limitations of our study are mainly due to the use of only one type of specimen collection swab and transport medium and by the lack of investigation of other environmental conditions, as high exposure temperatures, that could adversely affect sample integrity and therefore the testing results.

In conclusion, this study provides evidence that temperature and time of storage of nasopharyngeal swabs for SARS-CoV-2 testing have little impact on the reproducibility of results. However, great attention should be paid to nasopharyngeal swab sampling because, in cases of low viral load, this procedure can generate false-negative results. The experimental findings reported in the present study may be conducive to achieving standard operating procedures for the molecular diagnosis of COVID-19.

Research funding: None declared.

Author contributions: All authors have accepted responsibility for the entire content of this manuscript and approved its submission.

Competing interests: Authors state no conflict of interest. Informed consent: Informed consent was obtained from all individuals included in this study.

Ethical approval: Research involving human subjects complied with all relevant national regulations, institutional policies and is in accordance with the tenets of the Helsinki Declaration (as revised in 2013) and has been approved by the authors' Institutional Review Board (Local Ethic Committee Nr. 27444).

\section{References}

1. Siordia JA Jr. Epidemiology and clinical features of COVID-19: a review of current literature. J Clin Virol 2020. https://doi.org/10. 1016/j.jcv.2020.104357 [Epub ahead of print].

2. Leung K, Wu JT, Liu D, Leung GM. First-wave COVID-19 transmissibility and severity in China outside Hubei after control measures, and second-wave scenario planning: a modelling impact assessment. Lancet 2020;395:1382-93.

3. Sethuraman N, Jeremiah SS, Ryo A. Interpreting diagnostic tests for SARS-CoV-2. JAMA 2020. https://doi.org/10.1001/jama.2020. 8259 [Epub ahead of print].

4. Krammer F, Simon V. Serology assays to manage COVID-19 Science. 2020 May 15. pii: eabc1227. https://doi.org/10.1126/ science.abc1227 [Epub ahead of print].

5. Bohn MK, Lippi G, Horvath A, Sethi S, Koch D, Ferrari M, et al. Molecular, serological, and biochemical diagnosis and monitoring of COVID-19: IFCC taskforce evaluation of the latest evidence. Clin Chem Lab Med 2020;58:1037-52.
6. Zhao J, Yuan Q, Wang H, Liu W, Liao X, Su Y, et al. Antibody responses to SARS-CoV-2 in patients of novel coronavirus disease 2019. Clin Infect Dis 2020. https://doi.org/10.1093/cid/ ciaa344 [Epub ahead of print].

7. Padoan A, Cosma C, Sciacovelli L, Faggian D, Plebani M. Analytical performances of a chemiluminescence immunoassay for SARS-CoV-2 IgM/lgG and antibody kinetics. Clin Chem Lab Med 2020;58:1081-1088.

8. Yongchen Z, Shen H, Wang X, Shi X, Li Y, Yan J, et al. Different longitudinal patterns of nucleic acid and serology testing results based on disease severity of COVID-19 patients. Emerg Microbes Infect 2020;9:833-6.

9. Zhai P, Ding Y, Wu X, Long J, Zhong Y, Li Y. The epidemiology, diagnosis and treatment of COVID-19. Int J Antimicrob Agents 2020. https://doi.org/10.1016/j.ijantimicag.2020.105955 [Epub ahead of print].

10. Xiang F, Wang X, He X, Peng Z, Yang B, Zhang J, et al. Antibody Detection and Dynamic Characteristics in Patients with COVID-19. Clin Infect Dis 2020. https://doi.org/10.1093/cid/ciaa461 [Epub ahead of print].

11. World Health Organization (WHO). Laboratory testing for 2019 novel coronavirus (2019-nCoV) in suspected human cases. Available from: https://www.who.int/publicationsdetail/laboratory-testing-for-2019-novel-coronavirus-insuspected-human-cases-20200117 [Accessed 19 March 2020].

12. Chan JF, Yip CC, To KK, Tang TH, Wong SC, Leung KH, et al. Improved molecular diagnosis of COVID-19 by the novel, highly sensitive and specific COVID-19-RdRp/Hel realtime reverse transcription-PCR assay validated in vitro and with clinical specimens. J Clin Microbiol 2020;58: e00310-20.

13. Li Y, Yao L, Li J, Chen L, Song Y, Cai Z, Yang C. Stability issues of RTPCR testing of SARS-CoV-2 for hospitalized patients clinically diagnosed with COVID-19. J Med Virol 2020. https://doi.org/10. 1002/jmv.25786 [Epub ahead of print].

14. Wang W, Xu Y, Gao R, Lu R, Han K, Wu G, Tan W. Detection of SARS-CoV-2 in different types of clinical specimens. JAMA 2020. https://doi.org/10.1001/jama.2020.3786 [Epub ahead of print].

15. Plebani M. Errors in clinical laboratories or errors in laboratory medicine? Clin Chem Lab Med 2006;44:750-9.

16. Plebani $M$. The detection and prevention of errors in laboratory medicine. Ann Clin Biochem 2010;47:101-10.

17. Lippi G, Simundic AM, Plebani M. Potential preanalytical and analytical vulnerabilities in the laboratory diagnosis of coronavirus disease 2019 (COVID-19). Clin Chem Lab Med 2020;58:1070-1076. https://doi.org/10.1515/cclm-2020-0285 [Epub ahead of print].

18. Rodino KG, Espy MJ, Buckwalter SP, Walchak RC, Germer JJ, Fernholz E, et al. Evaluation of saline, phosphate buffered saline and minimum essential medium as potential alternatives to viral transport media for SARS-CoV-2 testing. J Clin Microbiol 2020. https://doi.org/10.1128/JCM.00590-20 [Epub ahead of print].

19. Radbel J, Jagpal S, Roy J, Brooks A, Tischfield J, Sheldon M, et al. Detection of severe acute respiratory syndrome Coronavirus 2 is comparable in clinical samples preserved in saline or viral transport medium. J Mol Diagn 2020 May 13:S1525-1578(20) 30323-8. https://doi.org/10.1016/j.jmoldx.2020.04.209 [Epub ahead of print]. 
20. Corman VM, Landt O, Kaiser M, Molenkamp R, Meijer A, Chu DKW, et al. Detection of 2019 novel coronavirus (2019-nCoV) by realtime RT-PCR. Euro Surveill 2020; https://doi.org/10.2807/15607917.ES.2020.25.3.2000045.

21. Yan Y, Chang L, Wang L. Laboratory testing of SARS-CoV, MERSCoV, and SARS-CoV-2 (2019-nCoV): Current status, challenges, and countermeasures. Rev Med Virol 2020. https://doi.org/10. 1002/rmv.2106 [Epub ahead of print].

22. Loens K, Bergs K, Ursi D, Goossens H, leven M. Evaluation of NucliSens easyMAG for automated nucleic acid extraction from various clinical specimens. J Clin Microbiol 2007;45: 421-5. 